\title{
ON THE LARGE DEVIATIONS THEOREM OF WEAKER TYPES
}

\author{
XINXING WU, XIONG WANG, AND GUANRONG CHEN
}

\begin{abstract}
In this paper, we introduce the concepts of the large deviations theorem of weaker types, i.e., type I, type I', type II, type II', type III, and type III', and present a systematic study of the ergodic and chaotic properties of dynamical systems satisfying the large deviations theorem of various types. Some characteristics of the ergodic measure are obtained and then applied to prove that every dynamical system satisfying the large deviations theorem of type I' is ergodic, which is equivalent to the large deviations theorem of type II' in this regard, and that every uniquely ergodic dynamical system restricted on its support satisfies the large deviations theorem. Moreover, we prove that every dynamical system satisfying the large deviations theorem of type III is an $E$-system. Finally, we show that a dynamical system satisfying the central limit theorem, introduced in [Y. Niu, Y. Wang, Statist. Probab. Lett., 80 (2010), 11801184], does not exist.
\end{abstract}

\section{INTRODUCTION}

A dynamical system is a pair $(X, T)$, where $X$ is a compact metric space with a metric $d$ and $T: X \rightarrow X$ is a continuous map. Sharkovsky's amazing discovery [27], as well as Li and Yorke's famous work which introduced the concept of 'chaos' known as LiYorke chaos today [20], have provoked the recent rapid advancement of research on discrete chaos theory. At the same time, many research works were devoted to the links between the topological and stochastic properties of deterministic dynamical systems.

The topological approach on chaoticity tries to describe the topological structure of a 'chaotic region' $[2,6,7,13,14,17,20,23]$. The essence of Li-Yorke chaos is the existence of uncountable scrambled sets. Another well-known definition of chaos was given by Devaney [7], according to which a continuous map $T$ is said to be chaotic in the sense of Devaney, if it satisfies the following three properties: With notation $\mathbb{N}=\{1,2, \ldots\}, \mathbb{Z}^{+}=\{0,1,2, \ldots\}$,

(1) $T$ is topologically transitive, i.e., for every pair of nonempty open sets $U, V \subset X$, there exists $n \in \mathbb{Z}^{+}$such that $T^{n}(U) \cap V \neq \varnothing$;

(2) The set of periodic points of $T$ is dense in $X$;

Date: July 6, 2018.

2010 Mathematics Subject Classification. Primary 54H20, 37B99; Secondary 54B20, 54H20, 37B20, 37B40.

Key words and phrases. Large deviations theorem, ergodic, transitivity, sensitivity, equicontinuous.

Xinxing Wu was supported by the Scientific Research Fund of Sichuan Provincial Education Department (No. 14ZB0007) and the scientific research starting project of SWPU.

Xiong Wang (Corresponding Author) was supported by the National Natural Science Foundation of China (No.11547117) and Seed Funding from Scientific and Technical Innovation Council of Shenzhen Government.

Guanrong Chen was supported by the Hong Kong Research Grants Council under GRF Grant CityU 11201414. 
(3) $T$ has sensitive dependence on initial conditions (briefly, is sensitive), i.e., there exists $\varepsilon>0$ such that for any $x \in X$ and any neighborhood $U$ of $x$, there exist $y \in U$ and $n \in \mathbb{Z}^{+}$satisfying $d\left(T^{n}(x), T^{n}(y)\right)>\varepsilon$.

Banks et al. [5] proved that every topologically transitive map whose periodic points are dense in $X$ has sensitive dependence on initial conditions, which implies that the above condition (3) is redundant, while Huang and Ye [14] showed that every topologically transitive map containing a periodic point is chaotic in the sense of Li-Yorke. Most importantly, sensitive dependence on initial conditions is widely understood as a key ingredient of chaos and was popularized by the meteorologist Lorenz, which is commonly known as the so-called 'butterfly effect'.

The stochastic approach is devoted to characterizing the dynamics of a deterministic dynamical system through its stochastic properties by using tools from ergodic theory, functional analysis and spectral theory $[4,21,22,26,28,29,30]$. Meanwhile, the links between the two approaches are being gradually studied $[1,11,12,18,19,25$, 31, 37, 40]. For example, Abraham et al. [1] gave some sufficient conditions (in terms of topology and ergodicity) on a measure-preserving dynamical system defined on a nontrivial metric space $(X, \mathcal{B}(X), \mu)$ endowed with a Borel probability measure, to ensure the sensitivity property or cofinite sensitivity property. In 2004, He et al. [12] proved that for a measure-preserving transformation $T$ on $(X, \mathcal{B}(X), \mu)$, if $\operatorname{supp}(\mu)=X$ and $T$ is weakly mixing, then $T$ has sensitive dependence on initial conditions and this also holds for measure-preserving semiflows. In 2006, Lardjane [18] complemented the main results in $[1,31,37]$ on the links between several topological and stochastic properties of dynamical systems and proved that if $\operatorname{supp}(\mu)=X$ and $T$ is mixing (notnecessarily measure-preserving), then $T$ has sensitive dependence on initial conditions.

It is commonly known that the large deviations theorem and the central limit theorem coming from probability theory are two of the most remarkable results in all fields of mathematics especially in probability and statistics. They were successfully applied to dynamical systems $[18,19,24,25,31,32,34,37]$. The former describes the oscillation of the time average $(1 / n) \sum_{i=0}^{n-1} \varphi \circ T^{i}(x)$ around the spatial average $\int_{X} \varphi \mathrm{d} \mu$ and the latter describes the rate of its convergence. In 2007, Gu [11] extended the results obtained by $\mathrm{Wu}$ et al. [31] and showed that a dynamical system satisfying the large deviations theorem is topologically ergodic. Moreover, if it is strongly topologically ergodic, then it has sensitive dependence on initial conditions (see [11, Theorem 3.1, Theorem 4.1]). Lately, Niu [24] proved that a dynamical system satisfying the large deviations theorem has an equicontinuous point if and only if it is both minimal and equicontinuous. Then, $\mathrm{Li}$ [19] introduced the concept of ergodic sensitivity, which is a stronger form of sensitivity, and showed that a strongly topologically ergodic system satisfying the large deviations theorem is ergodically sensitive. More recently, we [34] proved that a dynamical system satisfying the large deviations theorem is ergodic.

Based on the results in $[11,18,19,24,25,34]$, our objective here is to use the methods of ergodic theory and topological dynamics to further investigate the relations between the large deviations theorem, the ergodic properties and the chaotic behaviors of dynamical systems. 


\section{PReliminaries}

2.1. Topological dynamics. For $U, V \subset X$, define the return time set from $U$ to $V$ as $N(U, V)=\left\{n \in \mathbb{Z}^{+}: T^{n}(U) \cap V \neq \varnothing\right\}$. In particular, $N(x, V)=\left\{n \in \mathbb{Z}^{+}: T^{n}(x) \in V\right\}$ for $x \in X$.

Let $\mathcal{P}$ be the collection of all subsets of $\mathbb{Z}^{+}$. A collection $\mathscr{F} \subset \mathcal{P}$ is called a Furstenberg family if it is hereditary upwards, i.e., $F_{1} \subset F_{2}$ and $F_{1} \in \mathscr{F}$ imply $F_{2} \in \mathscr{F}$. A family $\mathscr{F}$ is proper if it is a proper subset of $\mathcal{P}$, i.e., neither empty nor the whole $\mathcal{P}$. It is easy to see that $\mathscr{F}$ is proper if and only if $\mathbb{Z}^{+} \in \mathscr{F}$ and $\varnothing \notin \mathscr{F}$. All the families considered below are assumed to be proper. For a Furstenberg family $\mathscr{F}$, denote $\Delta(\mathscr{F})=\{F-F: F \in \mathscr{F}\}$, where $F-F=\left\{i-j \in \mathbb{Z}^{+}: i, j \in F\right\}$.

For $A \subset \mathbb{Z}^{+}$, define

$$
\bar{d}(A)=\limsup _{n \rightarrow+\infty} \frac{1}{n}|A \cap[0, n-1]| \text { and } \underline{d}(A)=\liminf _{n \rightarrow+\infty} \frac{1}{n}|A \cap[0, n-1]| .
$$

Then, $\bar{d}(A)$ and $\underline{d}(A)$ are the upper density and the lower density of $A$, respectively.

Similarly, define the upper Banach density and the lower Banach density of $A$ as

$$
\mathrm{BD}^{*}(A)=\limsup _{|I| \rightarrow+\infty} \frac{|A \cap I|}{|I|} \text { and } \mathrm{BD}_{*}(A)=\liminf _{|I| \rightarrow+\infty} \frac{|A \cap I|}{|I|},
$$

where $I$ is over all non-empty finite intervals of $\mathbb{Z}^{+}$.

A subset $S$ of $\mathbb{Z}_{+}$is syndetic if it has a bounded gap, i.e., if there is $N \in \mathbb{N}$ such that $\{i, i+1, \ldots, i+N\} \cap S \neq \emptyset$ for every $i \in \mathbb{Z}^{+} ; S$ is thick if it contains arbitrarily long runs of positive integers, i.e., for every $n \in \mathbb{N}$ there exists some $a_{n} \in \mathbb{Z}^{+}$such that $\left\{a_{n}, a_{n}+1, \ldots, a_{n}+n\right\} \subset S$. The set of all thick subsets of $\mathbb{Z}^{+}$, all syndetic subsets of $\mathbb{Z}^{+}$, all subsets of $\mathbb{Z}^{+}$with positive upper density, all subsets of $\mathbb{Z}^{+}$with upper density equal to 1 , and all subsets of $\mathbb{Z}^{+}$with positive upper Banach density, are denoted by $\mathscr{F}_{t}$, $\mathscr{F}_{s}, \mathscr{F}_{\text {pud }}, \mathscr{F}_{\text {ud } 1}$, and $\mathscr{F}_{\text {pubd }}$, respectively. Clearly, all of them are Furstenberg families.

For a Furstenberg family $\mathscr{F}$, a dynamical system is called $\mathscr{F}$-transitive if $N(U, V) \in$ $\mathscr{F}$ for every pair of nonempty open subsets $U, V \subset X$. The $\mathscr{F}_{\text {pud }}$-transitivity and $\mathscr{F}_{u d 1^{-}}$ transitivity are called topological ergodicity and strongly topological ergodicity respectively in $[11,19]$. Clearly, $\mathscr{F}_{s}$-transitivity is stronger that $\mathscr{F}_{\text {pud }}$-transitivity.

A dynamical system $(X, T)$ is totally transitive if $\left(X, T^{n}\right)$ is transitive for each $n \in \mathbb{N}$; and it is (topologically) weakly mixing if $(X \times X, T \times T)$ is transitive. It is well known that $(X, T)$ is weakly mixing if and only if it is $\mathscr{F}_{t}$-transitive (see $\left.[8,9]\right)$. An $x \in X$ is $a$ transitive point if its orbital closure $\overline{\operatorname{orb}(x, T)}=X$. Let $\operatorname{Trans}(T)$ be the set of transitive points. Then, the orbit closure of a recurrent point is transitive.

A dynamical system $(X, T)$ is minimal if every orbit under $T$ is dense in $X$. It is easy to see that $(X, T)$ is a minimal system if and only if $X$ has no proper, nonempty, closed invariant subset. A point $x \in X$ is called an equicontinuity point of $T$ if, for any $\varepsilon>0$, there exists $\delta>0$ such that for any $y \in X$ with $d(x, y)<\delta$ and any $n \in \mathbb{Z}^{+}$, one has $d\left(T^{n}(x), T^{n}(y)\right)<\varepsilon$. A dynamical system $(X, T)$ is equicontinuous if every $x \in X$ is an equicontinuous point of $T ;(X, T)$ is almost equicontinuous if it is a transitive dynamical system admitting an equicontinuity point. By compactness, it can be verified that $(X, T)$ is equicontinuous when the sequence $\left\{T^{n}: n \in \mathbb{Z}^{+}\right\}$is uniformly equicontinuous.

For $U \subset X$ and $\varepsilon>0$, let

$$
N(U, \varepsilon)=\left\{n \in \mathbb{Z}^{+}: \operatorname{diam} T^{n}(U)>\varepsilon\right\} .
$$


It is easy to see that a dynamical system $(X, T)$ is sensitive if and only if there exists $\varepsilon>0$ such that, for any nonempty open subset $U \subset X, N(U, \varepsilon) \neq \varnothing$. For a dynamical system, Moothathu [23] initiated a preliminary study of stronger forms of sensitivity formulated in terms of some subsets of $\mathbb{Z}^{+}$, namely the syndetical sensitivity and cofinite sensitivity. Recently, Li [19] introduced the concept of ergodic sensitivity. According to Moothathu [23] and Li [19], a dynamical system $(X, T)$ is said to be

(1) ergodically sensitive if there exists $\varepsilon>0$ such that for any nonempty open subset $U \subset X, \bar{d}(N(U, \varepsilon))>0$;

(2) syndetically sensitive if there exists $\varepsilon>0$ such that for any nonempty open subset $U \subset X, N(U, \varepsilon)$ is syndetic;

(3) cofinitely sensitive if there exists $\varepsilon>0$ such that for any nonempty open subset $U \subset X, N(U, \varepsilon)$ is cofinite.

Clearly, cofinite sensitivity is stronger than syndetical sensitivity, which implies ergodic sensitivity. More results on sensitivity can be found in [16, 33, 36].

2.2. Probability measure. Let $\mathcal{B}(X)$ be the $\sigma$-algebra of Borel subsets of $X, M(X)$ the set of Borel probability measures on $(X, \mathcal{B}(X))$, and $M(X, T)$ the $T$-invariant ones. It is well known that $M(X)$ is a compact metrisable space in the weak*-topology, and $M(X, T)$ is a nonempty closed subset of $M(X)$. A measure-preserving transformation $T$ of a probability space $(X, \mathcal{B}(X), \mu)$ is called ergodic if the only members $B$ of $\mathcal{B}(X)$ with $T^{-1}(B)=B$ satisfy $\mu(B)=0$ or $\mu(B)=1$. A probability measure $\mu \in M(X, T)$ is called ergodic if the measure-preserving transformation $T$ of the measure space $(X, \mathcal{B}(X), \mu)$ is ergodic. Let $E(X, T)$ be the set of all ergodic measures in $M(X, T)$. If $M(X, T)$ consists of a single point, then $(X, T)$ is said to be uniquely ergodic.

For any $\mu \in M(X)$, the set $\{x \in X: \mu(U)>0$ for any neighborhood $U$ of $x\}$ is called the support of $\mu$, denoted by $\operatorname{supp}(\mu)$. As every Borel probability measure $\mu$ is regular (see [30, Theorem 6.1]), it is easy to see that $\mu(\operatorname{supp}(\mu))=1$ and $\operatorname{supp}\left(\left.\mu\right|_{\mathcal{B}(\operatorname{supp}(\mu))}\right)=$ $\operatorname{supp}(\mu)$.

Let $C(X)$ denote the Banach space of continuous complex-valued functions on $X$ with the supremum norm $\|\cdot\|$ and call each element of $C(X)$ an observable.

Lemma 2.1. [30, pp. 149] The following statements are equivalent:

(1) $\mu_{n} \rightarrow \mu$ in the weak*-topology.

(2) For each $\varphi \in C(X), \int_{X} \varphi \mathrm{d} \mu_{n} \rightarrow \int_{X} \varphi \mathrm{d} \mu$ as $n \rightarrow+\infty$.

(3) For each closed subset $F \subset X$, $\lim _{X \sup _{n \rightarrow+\infty}} \mu_{n}(F) \leq \mu(F)$.

(4) For each open subset $U \subset X$, $\liminf _{n \rightarrow+\infty} \mu_{n}(U) \geq \mu(U)$.

For $x \in X$, let $\delta_{x} \in M(X)$ denote the Dirac point measure of $x$, defined by

$$
\delta_{x}(A)= \begin{cases}1, & x \in A \\ 0, & x \notin A .\end{cases}
$$

For the ergodic measure, the following result is well known.

Lemma 2.2. Let $(X, T)$ be a dynamical system and $\mu \in M(X, T)$. Then,

(1) $\operatorname{supp}(\mu)$ is a nonempty, closed, invariant subset of $X$.

(2) If $\mu$ is ergodic, then $(\operatorname{supp}(\mu), \mathcal{B}(\operatorname{supp}(\mu)), T, \mu)$ is ergodic and transitive.

(3) If $T$ is uniquely ergodic, then $(\operatorname{supp}(\mu), T)$ is uniquely ergodic and minimal. 
A dynamical system $(X, T)$ is called an $E$-system if it is topologically transitive and there exists an invariant measure $\mu$ with a full support, i.e., $\operatorname{supp}(\mu)=X$. It is well known that every minimal system is an $E$-system, and every $E$-system is $\mathscr{F}_{s}$-transitive (see [10, Theorem 4.4]).

The main concern in the stochastic analysis of a deterministic dynamical system is how to describe the oscillations of the finite-time average

$$
\frac{1}{n} \sum_{i=0}^{n-1} \varphi \circ T^{i}(x)
$$

around their expected value $\int_{X} \varphi \mathrm{d} \mu$, where $\varphi$ is an observable, i.e., $\varphi \in C(X)$. The Birkhoff Ergodic Theorem indicates that for an ergodic measure-preserving transformation $T$ and for any $\varphi \in L^{1}(\mu)$,

$$
\lim _{n \rightarrow+\infty} \frac{1}{n} \sum_{i=0}^{n-1} \varphi \circ T^{i}(x)=\int_{X} \varphi \mathrm{d} \mu, \text { a.e. }
$$

Birkhoff Ergodic Theorem. Suppose that $T$ is a measure-preserving transformation of a probability space $(X, \mathcal{B}(X), \mu)$ and $\varphi \in L^{1}(\mu)$. Then, $(1 / n) \sum_{i=0}^{n-1} \varphi \circ T^{i}(x)$ converges a.e. to a limit function $\varphi^{*} \in L^{1}(\mu)$ such that $\varphi^{*} \circ T=\varphi^{*}$ and $\int_{X} \varphi^{*} \mathrm{~d} \mu=\int_{X} \varphi \mathrm{d} \mu$.

When $T$ is uniquely ergodic, the following result shows that it admits much stronger properties of these ergodic averages (see [30, Theorem 6.19]).

Lemma 2.3. [30, Theorem 6.19] Let $(X, T)$ be a dynamical system. The following statements are equivalent:

(1) For every $\varphi \in C(X),(1 / n) \sum_{i=0}^{n-1} \varphi \circ T^{i}(x)$ converges uniformly to a constant.

(2) For every $\varphi \in C(X),(1 / n) \sum_{i=0}^{n-1} \varphi \circ T^{i}(x)$ converges pointwise to a constant.

(3) There exists $\mu \in M(X, T)$ such that, for all $\varphi \in C(X)$ and all $x \in X$,

$$
\lim _{n \rightarrow+\infty} \frac{1}{n} \sum_{i=0}^{n-1} \varphi \circ T^{i}(x)=\int_{X} \varphi \mathrm{d} \mu .
$$

(4) $T$ is uniquely ergodic.

Meanwhile, by the Birkhoff Ergodic Theorem, it can be verified that a measurepreserving transformation $T$ of a probability space $(X, \mathcal{B}(X, \mu)$ is ergodic if and only if for any $A, B \in \mathcal{B}(X)$,

$$
\lim _{n \rightarrow+\infty} \frac{1}{n} \sum_{i=0}^{n-1} \mu\left(A \cap T^{-i}(B)\right)=\mu(A) \mu(B) .
$$

2.3. The large deviations theorem. First, recall the original large deviations theorem in classic probability theory.

Large deviations theorem. Let $X_{0}, X_{1}, \ldots$ be independent identically distributed random variables taking values in $\mathbb{R}$, with average $\bar{X}=E\left(X_{n}\right)<+\infty$, variance $\sigma^{2}=$ $E\left(\left(X_{n}-\bar{X}\right)^{2}\right) \in(0,+\infty)$, and $E\left(e^{t X_{n}}\right) \in(0,+\infty)$ for every $t \in \mathbb{R}$. Then, for any $\varepsilon>0$, the probability $\mathbf{P}(n, \varepsilon)$ of

$$
\left|\frac{1}{n} \sum_{i=0}^{n-1}\left(X_{i}-\bar{X}\right)\right|>\varepsilon
$$


converges to zero exponentially as $n \rightarrow+\infty$, in the sense that

$$
\limsup _{n \rightarrow+\infty} \frac{1}{n} \log \mathbf{P}(n, \varepsilon)<0 \text {. }
$$

Following the Birkhoff Ergodic Theorem and this large deviations theorem, Wu et al. [31] introduced the large deviations theorem for dynamical systems.

Let $(X, T)$ be a dynamical system and $\mu \in M(X)$. An observable $\varphi$ is said to satisfy the large deviations theorem for $(X, \mathcal{B}(X), T, \mu)$, or simply $(T, \mu)$, if for any $\varepsilon>0$ there exists $h(\varepsilon)>0$ such that

$$
\mu\left(\left\{x \in X:\left|\frac{1}{n} \sum_{i=0}^{n-1} \varphi \circ T^{i}(x)-\int_{X} \varphi \mathrm{d} \mu\right|>\varepsilon\right\}\right) \leq e^{-n h(\varepsilon)}
$$

for all sufficiently large $n \in \mathbb{N}$. According to Wu et al. [31], a dynamical system $(X, \mathcal{B}(X), T, \mu)$ or $(T, \mu)$ is said to satisfy the large deviations theorem if every observable satisfies the large deviations theorem for $(T, \mu)$ and $\operatorname{supp}(\mu)=X$.

To extend the large deviations theorem (LDT) modifying the convergence in (2.1), we now introduce some concepts of large deviations theorem of weaker forms. We say that $(T, \mu)$ satisfies

(1) the large deviations theorem of weak form (WLDT) if, every observable satisfies the large deviations theorem.

(2) the large deviations theorem of type I' (LDT-I') if, for every observable $\varphi$ and any $\varepsilon>0$,

$$
\sum_{n=1}^{+\infty} \mu\left(\left\{x \in X:\left|\frac{1}{n} \sum_{i=0}^{n-1} \varphi \circ T^{i}(x)-\int_{X} \varphi \mathrm{d} \mu\right|>\varepsilon\right\}\right)<+\infty
$$

(3) the large deviations theorem of type II' (LDT-II') if, for every observable $\varphi$ and any $\varepsilon>0$,

$$
\lim _{n \rightarrow+\infty} \mu\left(\left\{x \in X:\left|\frac{1}{n} \sum_{i=0}^{n-1} \varphi \circ T^{i}(x)-\int_{X} \varphi \mathrm{d} \mu\right|>\varepsilon\right\}\right)=0
$$

(4) the large deviations theorem of type III' (LDT-III') if, for every observable $\varphi$ and any $\varepsilon>0$,

$$
\liminf _{n \rightarrow+\infty} \mu\left(\left\{x \in X:\left|\frac{1}{n} \sum_{i=0}^{n-1} \varphi \circ T^{i}(x)-\int_{X} \varphi \mathrm{d} \mu\right|>\varepsilon\right\}\right)=0 .
$$

If $(T, \mu)$ satisfies LDT-I' (resp., LDT-II', LDT-III') and $\operatorname{supp}(\mu)=1$, then we say that $(T, \mu)$ satisfies the large deviations theorem of type I (LDT-I) (resp., type II (LDT-II), type III (LDT-III)).

Clearly,

$$
\text { LDT } \Rightarrow \text { LDT-I } \Rightarrow \text { LDT-II } \Rightarrow \text { LDT-III, }
$$

and

$$
\text { WLDT } \Rightarrow \text { LDT-I' } \Rightarrow \text { LDT-II' } \Rightarrow \text { LDT-III'. }
$$

Meanwhile, it can be verified that every trivial dynamical system satisfies LDT, and that $(X, \mathcal{B}(X), T, \mu)$ satisfies LDT-I' (resp., LDT-II', LDT-III') if and only if $(\operatorname{supp}(\mu), \mathcal{B}(\operatorname{supp}(\mu)), T, \mu)$ satisfies LDT-I (resp., LDT-II, LDT-III). We obtain a surprising result (see Theorem 3.3), however, which shows that LDT-II' is equivalent to the ergodicity. 
The following example shows that ergodicity, LDT, WLDT, LDT-I, LDT-I', LDT-II, LDT-II', LDT-III, and LDT-III' are not preserved under iterations. This example also shows that LDT does not guarantee the weakly mixing property. Let $\mathscr{P}_{1}$ be the set of all above listed properties.

Example 2.1. Let $X=\left\{a_{1}, a_{2}\right\}$ be any two distinct points with a discrete metric satisfying $\mu\left(a_{1}\right)=\mu\left(a_{2}\right)=1 / 2$. Define $T: X \rightarrow X$ as $T\left(a_{1}\right)=a_{2}$ and $T\left(a_{2}\right)=a_{1}$. It is easy to see that, for each $\mathrm{P} \in \mathscr{P}_{1}, T$ satisfies $\mathrm{P}$, but $T^{2}$ does not.

In [35], we proved that if there exists $n \in \mathbb{N}$ such that $\left(T^{n}, \mu\right)$ satisfies LDT, then $(T, \mu)$ satisfies LDT. Similarly, it can be verified that this also holds for all properties in $\mathscr{P}_{1}$, as summarized below.

Theorem 2.1. Let $(X, T)$ be a dynamical system, $\mu \in M(X, T)$, and $\mathrm{P} \in \mathscr{P}_{1}$. If there exists $n \in \mathbb{N}$ such that $\left(T^{n}, \mu\right)$ satisfies $\mathrm{P}$, then $(T, \mu)$ satisfies $\mathrm{P}$.

2.4. The central limit theorem. Recently, Niu and Wang [25] applied the central limit theorems in probability theory to dynamical systems and found certain relations between the central limit theorem and some chaotic properties.

An observable $\varphi$ is said to satisfy the Central Limit Theorem in the sense of Niu and Wang for $(T, \mu)$, if there exists $\sigma>0$ such that for every interval $A \subset \mathbb{R}$,

$$
\lim _{n \rightarrow+\infty} \mu\left(\left\{x \in X: \frac{1}{\sqrt{n}} \sum_{i=0}^{n-1}\left(\varphi \circ T^{i}(x)-\int_{X} \varphi \mathrm{d} \mu\right) \in A\right\}\right)=\frac{1}{\sigma \sqrt{2 \pi}} \int_{A} e^{-t^{2} /\left(2 \sigma^{2}\right)} \mathrm{d} t .
$$

If every observable satisfies the central limit theorem for $(T, \mu)$ and $\operatorname{supp}(\mu)=X$, it is said that $(T, \mu)$ satisfies the central limit theorem.

At the end of this paper, however, we will show that a dynamical system satisfying the Central Limit Theorem in the sense of Niu and Wang actually does not exist (see Theorem 6.1).

\section{THE LARGE DEVIATIONS THEOREM AND ERGODICITY}

In this section, some ergodic properties on dynamical systems satisfying LDT are obtained. The new results show that LDT-I' $\Rightarrow$ LDT-II' $\Leftrightarrow$ ergodicity.

The following lemma gives some characteristics to the ergodic measure.

Lemma 3.1. Let $(X, T)$ be a dynamical system and $\mu \in M(X)$. Then, the following statements are equivalent:

(1) $\mu$ is ergodic.

(2) There exists $Y \in \mathcal{B}(X)$ with $\mu(Y)=1$ such that, for all $y \in Y$,

$$
\frac{1}{n} \sum_{i=0}^{n-1} \delta_{T^{i}(y)} \rightarrow \mu
$$

(3) There exists $Y \in \mathcal{B}(X)$ with $\mu(Y)=1$ such that, for all $y \in Y$ and all $\varphi \in C(X)$,

$$
\lim _{n \rightarrow \infty} \frac{1}{n} \sum_{i=0}^{n-1} \varphi \circ T^{i}(y)=\int_{X} \varphi \mathrm{d} \mu .
$$


(4) For any $\varphi \in C(X)$ and any $\varepsilon>0$,

$$
\lim _{k \rightarrow \infty} \mu\left(\bigcup_{n=k}^{+\infty}\left\{x \in X:\left|\frac{1}{n} \sum_{i=0}^{n-1} \varphi \circ T^{i}(x)-\int_{X} \varphi \mathrm{d} \mu\right|>\varepsilon\right\}\right)=0 .
$$

(5) For any $\varphi \in C(X),(1 / n) \sum_{i=0}^{n-1} \varphi \circ T^{i}(x) \rightarrow \int_{X} \varphi \mathrm{d} \mu$ a.e.

Proof. (2) $\Leftrightarrow$ (3) follows from Lemma 2.1. In view of [30, Theorem 6.14], it suffices to check that (3) $\Leftrightarrow$ (4), and that $\mu \in M(X, T)$ under the assumption of (3).

$(3) \Rightarrow(4)$. Given any fixed $\varphi \in C(X)$, one has

$$
\begin{aligned}
X \backslash Y \supset \operatorname{Div}(\varphi) & :=\left\{x \in X: \frac{1}{n} \sum_{i=0}^{n-1} \varphi \circ T^{i}(x) \nrightarrow \int_{X} \varphi \mathrm{d} \mu\right\} \\
& =\bigcup_{n=1}^{+\infty} \bigcap_{m=1}^{+\infty} \bigcup_{k=m}^{+\infty}\left\{x \in X:\left|\frac{1}{k} \sum_{i=0}^{k-1} \varphi \circ T^{i}(x)-\int_{X} \varphi \mathrm{d} \mu\right|>\frac{1}{n}\right\} .
\end{aligned}
$$

Then, $\mu(\operatorname{Div}(\varphi))=0$. So, for any $n \in \mathbb{N}$,

$$
\lim _{m \rightarrow+\infty}\left(\bigcup_{k=m}^{+\infty}\left\{x \in X:\left|\frac{1}{k} \sum_{i=0}^{k-1} \varphi \circ T^{i}(x)-\int_{X} \varphi \mathrm{d} \mu\right|>\frac{1}{n}\right\}\right)=0 .
$$

This implies that, for any $\varepsilon>0$,

$$
\lim _{m \rightarrow+\infty}\left(\bigcup_{k=m}^{+\infty}\left\{x \in X:\left|\frac{1}{k} \sum_{i=0}^{k-1} \varphi \circ T^{i}(x)-\int_{X} \varphi \mathrm{d} \mu\right|>\varepsilon\right\}\right)=0 .
$$

(4) $\Rightarrow(3)$. Given any fixed $\varphi \in C(X)$, condition (4) implies that, for any $n \in \mathbb{N}$,

$$
\begin{aligned}
& \mu\left(\bigcap_{m=1}^{+\infty} \bigcup_{k=m}^{+\infty}\left\{x \in X:\left|\frac{1}{k} \sum_{i=0}^{k-1} \varphi \circ T^{i}(x)-\int_{X} \varphi \mathrm{d} \mu\right|>\frac{1}{n}\right\}\right) \\
= & \lim _{m \rightarrow \infty} \mu\left(\bigcup_{k=m}^{+\infty}\left\{x \in X:\left|\frac{1}{k} \sum_{i=0}^{k-1} \varphi \circ T^{i}(x)-\int_{X} \varphi \mathrm{d} \mu\right|>\frac{1}{n}\right\}\right)=0 .
\end{aligned}
$$

Combining this with

$$
\begin{aligned}
\operatorname{Con}(\varphi) & :=\left\{x \in X: \frac{1}{n} \sum_{i=0}^{n-1} \varphi \circ T^{i}(x) \rightarrow \int_{X} \varphi \mathrm{d} \mu\right\} \\
& =\bigcap_{n=1}^{+\infty} \bigcup_{m=1}^{+\infty} \bigcap_{k=m}^{+\infty}\left\{x \in X:\left|\frac{1}{k} \sum_{i=0}^{k-1} \varphi \circ T^{i}(x)-\int_{X} \varphi \mathrm{d} \mu\right|<\frac{1}{n}\right\},
\end{aligned}
$$

it follows that $\mu(\operatorname{Con}(\varphi))=1$. Choose a countable dense subset $\left\{\varphi_{k}\right\}_{k=1}^{+\infty}$ of $C(X)$ and take $Y=\bigcap_{k=1}^{+\infty} \operatorname{Con}\left(\varphi_{k}\right)$. Then, $\mu(Y)=1$ and, for any $y \in Y$ and any $k \in \mathbb{N}$,

$$
\lim _{n \rightarrow+\infty} \frac{1}{n} \sum_{i=0}^{n-1} \varphi_{k} \circ T^{i}(y)=\int_{X} \varphi_{k} \mathrm{~d} \mu .
$$

The result follows from approximating a given $\varphi \in C(X)$ by members of $\left\{\varphi_{k}\right\}_{k=1}^{+\infty}$.

Applying (3.1), it is easy to see (4) $\Leftrightarrow$ (5). 
Finally, according to the proof of the Krylov-Bogolioubov Theorem (see [30, Theorem 6.9]), it is easy to see that under the assumption of (3), $\mu$ is $T$-invariant, i.e., $\mu \in M(X, T)$. Its proof is included here for completeness.

In fact, for any given $\varphi \in C(X)$ and $y \in Y$, noting that $\varphi \circ T \in C(X)$, condition (3) implies that $(1 / n) \sum_{i=0}^{n-1} \varphi \circ T^{i}(y) \rightarrow \int_{X} \varphi \mathrm{d} \mu$ and $(1 / n) \sum_{i=0}^{n-1}(\varphi \circ T) \circ T^{i}(y) \rightarrow \int_{X} \varphi \circ T \mathrm{~d} \mu$. Combining this with $\left|(1 / n) \sum_{i=0}^{n-1} \varphi \circ T^{i}(y)-(1 / n) \sum_{i=0}^{n-1}(\varphi \circ T) \circ T^{i}(y)\right| \leq 2\|\varphi\| / n$, it follows that

$$
\int_{X} \varphi \mathrm{d} \mu-\int_{X} \varphi \circ T \mathrm{~d} \mu=\lim _{n \rightarrow+\infty} \frac{1}{n} \sum_{i=0}^{n-1} \varphi \circ T^{i}(y)-\lim _{n \rightarrow+\infty} \frac{1}{n} \sum_{i=0}^{n-1}(\varphi \circ T) \circ T^{i}(y)=0 .
$$

The result is now implied by [30, Theorem 6.8].

Remark 1. (1) It is noticeable that [30, Theorem 6.14] shows that Lemma 3.1 (1) is equivalent to Lemma 3.1 (2), under the hypothesis of $\mu \in M(X, T)$.

(2) By Lemma 3.1 (3), it is easy to see that $\mu \in M(X, T)$ is ergodic if and only if $\mu\left(\left\{x \in X: \lim _{n \rightarrow+\infty}(1 / n) \sum_{i=0}^{n-1} \varphi \circ T^{i}(x)=\int_{X} \varphi \mathrm{d} \mu, \forall \varphi \in C(X)\right\}\right)=1$.

Theorem 3.1. Let $(X, T)$ be a dynamical system and $\mu \in M(X)$. If $(T, \mu)$ satisfies LDT-I', then $\mu$ is ergodic.

Proof. The result follows from Lemma 3.1 and the fact that

$$
\begin{aligned}
& \mu\left(\bigcup_{n=k}^{+\infty}\left\{x \in X:\left|\frac{1}{n} \sum_{i=0}^{n-1} \varphi \circ T^{i}(x)-\int_{X} \varphi \mathrm{d} \mu\right|>\varepsilon\right\}\right) \\
\leq & \sum_{n=k}^{+\infty} \mu\left(\left\{x \in X:\left|\frac{1}{n} \sum_{i=0}^{n-1} \varphi \circ T^{i}(x)-\int_{X} \varphi \mathrm{d} \mu\right|>\varepsilon\right\}\right) .
\end{aligned}
$$

Because LDT is stronger than LDT-I', Theorem 3.1 immediately generates the following corollaries.

Corollary 3.1. If $(T, \mu)$ satisfies WLDT, then $\mu$ ergodic.

Corollary 3.2. If $(T, \mu)$ satisfies LDT or LDT-I, then $\mu$ is an ergodic measure with a full support.

Corollary 3.3. [11, Theorem 3.1] If $(T, \mu)$ satisfies LDT, then $T$ is topologically ergodic.

Proof. Since every ergodic dynamical system with full support is $\mathscr{F}_{s}$-transitive, this follows by Corollary 3.2.

Theorem 3.2. Let $(X, T)$ be a uniquely ergodic dynamical system and $\mu \in E(X, T)$. Then,

(1) $(T, \mu)$ satisfies WLDT.

(2) $\left(\left.T\right|_{\operatorname{supp}(\mu)}, \mu\right)$ satisfies $L D T$.

Proof. The unique ergodicity of $(X, T)$, together with Lemma 2.3, implies that for every $\varphi \in C(X),(1 / n) \sum_{i=0}^{n-1} \varphi \circ T^{i}(x)$ converges uniformly to $\int_{X} \varphi \mathrm{d} \mu$. This means that for any $\varepsilon>0$, there exists $N \in \mathbb{N}$ such that for all $x \in X,\left|(1 / n) \sum_{i=0}^{n-1} \varphi \circ T^{i}(x)-\int_{X} \varphi \mathrm{d} \mu\right|<\varepsilon$ holds for all $n \geq N$. So, $(T, \mu)$ satisfies WLDT.

(2) This follows by (1) and $\operatorname{supp}\left(\left.\mu\right|_{\mathcal{B}(\operatorname{supp}(\mu))}\right)=\operatorname{supp}(\mu)$. 
Theorem 3.2 with Lemma 2.2 leads to the following result.

Corollary 3.4. Let $(X, T)$ be a uniquely ergodic dynamical system and $\mu \in M(X)$. Then, the following statements are equivalent:

(1) $(T, \mu)$ satisfies LDT.

(2) $(T, \mu)$ satisfies LDT-I.

(3) $(T, \mu)$ satisfies LDT-II.

(4) $T$ is minimal and $\mu \in M(X, T)$.

Remark 2. Corollary 3.4 indicates that a nontrivial dynamical system satisfying LDT, which is sensitive or equicontinuous, indeed exists.

The following Proposition 3.1 shows that the probability measure $\mu$ of the pair $(T, \mu)$ satisfying LDT-II' is $T$-invariant. Theorem 3.3 gives an improved characteristic of LDT-II' which indicates that LDT-II' is equivalent to ergodicity.

Proposition 3.1. Let $(X, T)$ be a dynamical system and $\mu \in M(X)$. If $(T, \mu)$ satisfies LDT-II', then $\mu$ is T-invariant.

Proof. By [30, Theorem 6.8], it suffices to check that, for any $\varphi \in C(X), \int_{X} \varphi \mathrm{d} \mu=$ $\int_{X} \varphi \circ T \mathrm{~d} \mu$. Since $\varphi \circ T \in C(X)$ and $(T, \mu)$ satisfies LDT-II', it follows that, for any $M \in \mathbb{N}$

$$
\lim _{n \rightarrow+\infty} \mu\left(\left\{x \in X:\left|\frac{1}{n} \sum_{i=0}^{n-1} \varphi \circ T^{i}(x)-\int_{X} \varphi \mathrm{d} \mu\right| \leq \frac{1}{2 M}\right\}\right)=1
$$

and

$$
\lim _{n \rightarrow+\infty} \mu\left(\left\{x \in X:\left|\frac{1}{n} \sum_{i=0}^{n-1}(\varphi \circ T) \circ T^{i}(x)-\int_{X} \varphi \circ T \mathrm{~d} \mu\right| \leq \frac{1}{2 M}\right\}\right)=1 .
$$

Thus, there exists $N \in \mathbb{N}$ such that, for any $n \geq N$, there exists $x_{n} \in X$ satisfying $\left|(1 / n) \sum_{i=0}^{n-1} \varphi \circ T^{i}\left(x_{n}\right)-\int_{X} \varphi \mathrm{d} \mu\right| \leq 1 / 2 M$ and $\left|(1 / n) \sum_{i=0}^{n-1}(\varphi \circ T) \circ T^{i}\left(x_{n}\right)-\int_{X} \varphi \circ T \mathrm{~d} \mu\right| \leq$ $1 / 2 M$. This implies that

$$
\begin{aligned}
& \left|\int_{X} \varphi \mathrm{d} \mu-\int_{X} \varphi \circ T \mathrm{~d} \mu\right| \\
\leq & \left|\int_{X} \varphi \mathrm{d} \mu-\frac{1}{n} \sum_{i=0}^{n-1} \varphi \circ T^{i}\left(x_{n}\right)\right|+\left|\frac{1}{n} \sum_{i=0}^{n-1} \varphi \circ T^{i}\left(x_{n}\right)-\frac{1}{n} \sum_{i=0}^{n-1}(\varphi \circ T) \circ T^{i}\left(x_{n}\right)\right| \\
& +\left|\frac{1}{n} \sum_{i=0}^{n-1}(\varphi \circ T) \circ T^{i}\left(x_{n}\right)-\int_{X} \varphi \circ T \mathrm{~d} \mu\right| \\
\leq & \frac{1}{2 M}+\frac{2\|\varphi\|}{n}+\frac{1}{2 M}=\frac{1}{M}+\frac{2\|\varphi\|}{n} .
\end{aligned}
$$

So, $\int_{X} \varphi \mathrm{d} \mu=\int_{X} \varphi \circ T \mathrm{~d} \mu$.

Theorem 3.3. Let $(X, T)$ be a dynamical system and $\mu \in M(X)$. Then, $(T, \mu)$ satisfies LDT-II' if and only if $\mu$ is ergodic.

Proof. The sufficiency follows immediately from Lemma 3.1.

To prove the necessity, by Lemma 3.1, it suffices to check that, for any $\varphi \in C(X)$, $(1 / n) \sum_{i=0}^{n-1} \varphi \circ T^{i}(x) \rightarrow \int_{X} \varphi \mathrm{d} \mu$ a.e. 
Claim 1. For any $\varphi \in C(X)$, there exists an increasing sequence $\left\{L_{k}\right\}_{k=1}^{+\infty} \subset \mathbb{N}$ such that $\left(1 / L_{k}\right) \sum_{i=0}^{L_{k}-1} \varphi \circ T^{i}(x) \rightarrow \int_{X} \varphi \mathrm{d} \mu$ a.e.

Given any $N \in \mathbb{N}$, since $(T, \mu)$ satisfies LDT-II', one has

$$
\lim _{n \rightarrow+\infty} \mu\left(\Sigma(n, 1 / N):=\left\{x \in X:\left|\frac{1}{n} \sum_{i=0}^{n-1} \varphi \circ T^{i}(x)-\int_{X} \varphi \mathrm{d} \mu\right|>\frac{1}{N}\right\}\right)=0 .
$$

In particular, for $N=1$, there exists an increasing sequence $\left\{L_{k}^{(1)}\right\}_{k=1}^{+\infty}$ such that, for any $k \in \mathbb{N}, \mu\left(\Sigma\left(L_{k}^{(1)}, 1\right)\right) \leq 1 / 2^{k}$. Consider the sequence of numbers $\left\{\mu\left(\Sigma\left(L_{k}^{(1)}, 1 / 2\right)\right)\right\}_{k=1}^{+\infty}$, which converges to zero by the fact that $(T, \mu)$ satisfies LDT-II' and so has a subsequence $\left\{\mu\left(\Sigma\left(L_{k}^{(2)}, 1 / 2\right)\right)\right\}_{k=1}^{+\infty}$ such that for any $k \in \mathbb{N}, \mu\left(\Sigma\left(L_{k}^{(2)}, 1 / 2\right)\right) \leq 1 / 2^{k}$. Clearly, $\sum_{k=1}^{+\infty} \mu\left(\Sigma\left(L_{k}^{(2)}, 1\right)\right) \leq \sum_{k=1}^{+\infty} 1 / 2^{k}=1$. Proceed in this process and, for each $N \in \mathbb{N}$, obtain a subsequence $\left\{L_{k}^{(N)}\right\}_{k=1}^{+\infty} \subset \mathbb{N}$ such that $\left\{L_{k}^{(N)}\right\}_{k=1}^{+\infty} \subset\left\{L_{k}^{(N-1)}\right\}_{k=1}^{+\infty} \subset \cdots \subset\left\{L_{k}^{(2)}\right\}_{k=1}^{+\infty} \subset$ $\left\{L_{k}^{(1)}\right\}_{k=1}^{+\infty}$, so that $\sum_{k=1}^{+\infty} \mu\left(\Sigma\left(L_{k}^{(j)}, 1 / j\right)\right) \leq 1$ for $j=1, \ldots, N$. Choose the diagonal $\left\{L_{k}=L_{k}^{(k)}\right\}_{k=1}^{+\infty}$. It is easy to see that $\sum_{k=1}^{+\infty} \mu\left(\Sigma\left(L_{k}, 1 / j\right)\right) \leq 1$ holds for all $j=1,2, \ldots$. Combining this with

$$
\begin{aligned}
Q & :=\left\{x \in X: \frac{1}{L_{k}} \sum_{i=0}^{L_{k}-1} \varphi \circ T^{i}(x) \nrightarrow \int_{X} \varphi \mathrm{d} \mu\right\} \\
& =\bigcup_{n=1}^{+\infty} \bigcap_{m=1}^{+\infty} \bigcup_{k=m}^{+\infty}\left\{x \in X:\left|\frac{1}{L_{k}} \sum_{i=0}^{L_{k}-1} \varphi \circ T^{i}(x)-\int_{X} \varphi \mathrm{d} \mu\right|>\frac{1}{n}\right\},
\end{aligned}
$$

it follows that $\mu(Q)=0$. The proof of Claim 1 is thus completed.

Claim 2. For any $\varphi \in C(X),(1 / n) \sum_{i=0}^{n-1} \varphi \circ T^{i}(x) \rightarrow \int_{X} \varphi \mathrm{d} \mu$ a.e.

By the Birkhoff Ergodic Theorem, Proposition 3.1, and Claim 1, there exist $\varphi^{*} \in$ $L^{1}(\mu)$ (as $\left.\varphi \in C(X) \subset L^{1}(\mu)\right)$ and an increasing sequence $\left\{L_{k}\right\}_{k=1}^{+\infty}$ such that $(1 / n) \sum_{i=0}^{n-1} \varphi \circ$ $T^{i}(x) \rightarrow \varphi^{*}(x)$ a.e. and $\left(1 / L_{k}\right) \sum_{i=0}^{L_{k}-1} \varphi \circ T^{i}(x) \rightarrow \int_{X} \varphi \mathrm{d} \mu$ a.e. Take $\Sigma=\{x \in X:$ $\left.(1 / n) \sum_{i=0}^{n-1} \varphi \circ T^{i}(x) \rightarrow \varphi^{*}(x)\right\} \bigcap\left\{x \in X:\left(1 / L_{k}\right) \sum_{i=0}^{L_{k}-1} \varphi \circ T^{i}(x) \rightarrow \int_{X} \varphi \mathrm{d} \mu\right\}$. Clearly, $\mu(\Sigma)=1$ and, for any $x \in \Sigma$,

$$
\varphi^{*}(x)=\lim _{n \rightarrow+\infty} \frac{1}{n} \sum_{i=0}^{n-1} \varphi \circ T^{i}(x)=\lim _{k \rightarrow+\infty} \frac{1}{L_{k}} \sum_{i=0}^{L_{k}-1} \varphi \circ T^{i}(x)=\int_{X} \varphi \mathrm{d} \mu .
$$

This implies that $(1 / n) \sum_{i=0}^{n-1} \varphi \circ T^{i}(x) \rightarrow \int_{X} \varphi \mathrm{d} \mu$ a.e.

In the proof of Theorem 3.3, the diagonalization procedure works. We will give another proof of this result in Section 5 (see Lemma 5.1).

Corollary 3.5. Let $(X, T)$ be a dynamical system and $\mu \in M(X)$. If $(T, \mu)$ satisfies LDT, LDT-I or LDT-II, then $(X, T)$ is an E-system. In particular, $T$ is $\mathscr{F}_{s}$-transitive.

Proof. The result yields by Theorem 3.3. 


\section{THE LARGE DEVIATIONS THEOREM AND SENSITIVITY}

An interesting question about a dynamical system is when its orbits from nearby points start to separate after finite steps. This is also one of the most important features depicting the chaoticity of a system. This notion, referred to as the "butterfly effect", has been widely studied and is termed the sensitive dependence on initial conditions (briefly, sensitivity), introduced by Auslander and Yorke [3] and popularized by Devaney [7].

In [15], Huang and Ye proved that an almost equicontinuous $\mathscr{F}_{s}$-transitive system is minimal and equicontinuous. This, together with the Auslander-Yorke dichotomy Theorem (also see [2, Theorem 3.1]) and [23, Corollary 1], which states that for an $\mathscr{F}_{s}$-transitive system, sensitivity implies syndetical sensitivity, implies that every $\mathscr{F}_{s^{-}}$ transitive system is either syndetically sensitive or both minimal and equicontinuous. These with Corollary 3.5 lead to the following results.

Theorem 4.1. If $(X, T)$ is strongly topologically ergodic, then $T$ is weakly mixing. In particular, $T$ is sensitive.

Proof. It follows from the fact that the weakly mixing property is equivalent to the $\mathscr{F}_{t}$-transitivity and $\mathscr{F}_{t} \supset \mathscr{F}_{u d 1}$.

Theorem 4.2. If $(T, \mu)$ satisfies LDT-II and $T$ is sensitive, then $T$ is syndetically sensitive.

Theorem 4.2 implies [11, Theorem 4.1] immediately.

Corollary 4.1. If $(T, \mu)$ satisfies LDT-II and $T$ is strongly topologically ergodic, then $T$ is syndetically sensitive.

Corollary 4.2. [19, Theorem 3.1] If $(T, \mu)$ satisfies LDT and $T$ is strongly topologically ergodic, then $T$ is ergodically sensitive.

Corollary 4.3. If $(T, \mu)$ satisfies LDT-II, then $T$ is either syndetically sensitive or both minimal and equicontinuous.

For the $\mathscr{F}$-transitivity, we have the following result. Note that the proof is similar to [15, Theorem 4.6], but for completeness, a proof is provided here.

Theorem 4.3. Let $(X, T)$ be a dynamical system and let $\mathscr{F}$ be a Furstenberg family. If $(X, T)$ is $\mathscr{F}$-transitive and almost equicontinuous, then for any $x \in \operatorname{Trans}(T)$ and any neighbourhood $U$ of $x, N(x, U) \in \Delta(\mathscr{F})$.

Proof. Fix any $x \in \operatorname{Trans}(T)$ and any $\varepsilon>0$. Noting that $x$ is an equicontinuous point, it follows that there exists $0<\varepsilon_{1}<\varepsilon / 2$ such that, for any $y \in B\left(x, \varepsilon_{1}\right)$ and any $n \in \mathbb{Z}^{+}$, $d\left(T^{n}(x), T^{n}(y)\right)<\varepsilon / 2$. For any $n \in N\left(B\left(x, \varepsilon_{1}\right), B\left(x, \varepsilon_{1}\right)\right)$, there exists $y \in B\left(x, \varepsilon_{1}\right)$ such that $T^{n}(y) \in B\left(x, \varepsilon_{1}\right)$. Thus, $d\left(x, T^{n}(x)\right) \leq d(x, y)+d\left(T^{n}(x), T^{n}(y)\right)<\varepsilon$, i.e., $N\left(B\left(x, \varepsilon_{1}\right), B\left(x, \varepsilon_{1}\right)\right) \subset N(x, B(x, \varepsilon)) \in \mathscr{F}$. This, together with [38, Proposition 2.2], implies that for any neighbourhood $U$ of $x, N(U, U)=N(x, U)-N(x, U) \in \Delta(\mathscr{F})$. Repeating the proof above, one obtains that $N(x, U) \in \Delta(\mathscr{F})$.

Now, consider the non-sensitive dynamical systems satisfying LDT-II'.

Theorem 4.4. Let $(X, T)$ be a dynamical system and $(T, \mu)$ satisfy LDT-II'. If $(X, T)$ is not sensitive, then 
(1) $(\operatorname{supp}(\mu), T)$ is minimal and equicontinuous.

(2) For every observable $\varphi \in C(\operatorname{supp}(\mu))$,

$$
\lim _{n \rightarrow+\infty} \frac{1}{n} \sum_{i=0}^{n-1} \varphi \circ T^{i}(x)=\int_{\operatorname{supp}(\mu)} \varphi \mathrm{d} \mu, \quad \forall x \in \operatorname{supp}(\mu) .
$$

(3) $(\operatorname{supp}(\mu), T)$ is uniquely ergodic.

Proof. (1) It follows directly from Corollary 3.5 and Theorem 4.3.

(2) Suppose, on the contrary, that there exist an observable $\varphi \in C(\operatorname{supp}(\mu))$ and $x \in \operatorname{supp}(\mu)$ such that $(1 / n) \sum_{i=0}^{n-1} \varphi \circ T^{i}(x) \nrightarrow \int_{\operatorname{supp}(\mu)} \varphi \mathrm{d} \mu$, i.e.,

$$
\xi:=\limsup _{n \rightarrow+\infty} \frac{1}{n} \sum_{i=0}^{n-1} \varphi \circ T^{i}(x) \neq \int_{\operatorname{supp}(\mu)} \varphi \mathrm{d} \mu,
$$

or

$$
\eta:=\liminf _{n \rightarrow+\infty} \frac{1}{n} \sum_{i=0}^{n-1} \varphi \circ T^{i}(x) \neq \int_{\operatorname{supp}(\mu)} \varphi \mathrm{d} \mu .
$$

Without loss of generality, assume that $\xi>\int_{\operatorname{supp}(\mu)} \varphi \mathrm{d} \mu$, because the rest cases can be verified similarly. Then, there exists an increasing sequence $\left\{n_{k}\right\}_{k=1}^{+\infty} \subset \mathbb{N}$ such that, for any $k \in \mathbb{N}$,

$$
\frac{1}{n_{k}} \sum_{i=0}^{n_{k}-1} \varphi \circ T^{i}(x)>\frac{1}{4}\left(3 \xi+\int_{\operatorname{supp}(\mu)} \varphi \mathrm{d} \mu\right) .
$$

Since $\varphi$ is uniformly continuous, there exists $0<\delta_{1}<\left(\xi-\int_{\operatorname{supp}(\mu)} \varphi \mathrm{d} \mu\right) / 4$ such that, for any $x_{1}, x_{2} \in X$ with $d\left(x_{1}, x_{2}\right)<\delta_{1}$,

$$
\left|\varphi\left(x_{1}\right)-\varphi\left(x_{2}\right)\right|<\frac{1}{4}\left(\xi-\int_{\operatorname{supp}(\mu)} \varphi \mathrm{d} \mu\right) .
$$

The equicontinuity of $T$ implies that there exists $0<\delta<\delta_{1}$ such that, for any $y \in$ $B(x, \delta):=\{y \in \operatorname{supp}(\mu): d(x, y)<\delta\}$ and any $n \in \mathbb{Z}^{+}, d\left(T^{n}(x), T^{n}(y)\right)<\delta_{1}$. Combining this with (4.1) and (4.2), for any $y \in B(x, \delta)$ and any $k \in \mathbb{N}$, one has

$$
\begin{aligned}
\frac{1}{n_{k}} \sum_{i=0}^{n_{k}-1} \varphi \circ T^{i}(y) & =\frac{1}{n_{k}} \sum_{i=0}^{n_{k}-1} \varphi \circ T^{i}(x)-\frac{1}{n_{k}} \sum_{i=0}^{n_{k}-1}\left[\varphi \circ T^{i}(x)-\varphi \circ T^{i}(y)\right] \\
& \geq \frac{1}{n_{k}} \sum_{i=0}^{n_{k}-1} \varphi \circ T^{i}(x)-\frac{1}{n_{k}} \sum_{i=0}^{n_{k}-1}\left|\varphi \circ T^{i}(x)-\varphi \circ T^{i}(y)\right| \\
& >\frac{1}{4}\left(3 \xi+\int_{\operatorname{supp}(\mu)} \varphi \mathrm{d} \mu\right)-\frac{1}{4}\left(\xi-\int_{\operatorname{supp}(\mu)} \varphi \mathrm{d} \mu\right) \\
& =\frac{1}{2}\left(\xi+\int_{\operatorname{supp}(\mu)} \varphi \mathrm{d} \mu\right),
\end{aligned}
$$

i.e.,

$$
\frac{1}{n_{k}} \sum_{i=0}^{n_{k}-1} \varphi \circ T^{i}(y)-\int_{\operatorname{supp}(\mu)} \varphi \mathrm{d} \mu>\frac{1}{2}\left(\xi-\int_{\operatorname{supp}(\mu)} \varphi \mathrm{d} \mu\right)>0 .
$$


This implies that, for any $k \in \mathbb{N}$,

$\left\{z \in \operatorname{supp}(\mu):\left|\frac{1}{n_{k}} \sum_{i=0}^{n_{k}-1} \varphi \circ T^{i}(z)-\int_{\operatorname{supp}(\mu)} \varphi \mathrm{d} \mu\right|>\frac{1}{2}\left(\xi-\int_{\operatorname{supp}(\mu)} \varphi \mathrm{d} \mu\right)\right\} \supset B(x, \delta)$,

which is a contradiction since $\left(\left.T\right|_{\operatorname{supp}(\mu)}, \mu\right)$ satisfies LDT-II and $\mu(B(x, \delta))>0$.

(3) Based on Lemma 2.3, Proposition 3.1, and (2), this holds trivially.

Corollary 4.4. Let $T$ be a measure-preserving transformation of a probability space $(X, \mathcal{B}(X, \mu)$. Then, $(X, T)$ is a non-sensitive ergodic system with full support if and only if $T$ is minimal, equicontinuous, and uniquely ergodic.

Proof. It follows immediately from Corollary 5.2, Theorem 3.3, and Theorem 4.4.

\section{THE LARGE DEVIATIONS THEOREM OF TYPE III AND TRANSITIVITY}

This section studies the transitivity of dynamical systems satisfying LDT-III.

Theorem 5.1. Let $(X, T)$ be a dynamical system and $\mu \in M(X)$. If $(T, \mu)$ satisfies LDT-III', then:

(1) For any nonempty open subset $U \subset X$ with $\mu(U)>0$, there exists $x \in U$ such that $\bar{d}(N(x, U))>0$.

(2) For any nonempty open subsets $U, V \subset X$ with $\mu(U) \mu(V)>0, N(U, V) \neq \varnothing$.

Proof. (1) Suppose, on the contrary, that (1) does not hold. Then, there exists a nonempty open set $U \subset X$ with $\mu(U)>0$ such that, for any $x \in U, \bar{d}(N(x, U))=0$, i.e.,

$$
\lim _{n \rightarrow+\infty} \frac{1}{n}\left|\left\{0 \leq i<n: T^{i}(x) \in U\right\}\right|=0 .
$$

Take a nonempty open subset $V \subset U$ satisfying $\bar{V} \subset U$. Clearly, both $\bar{V}$ and $X \backslash U$ are nonempty closed subsets of $X$, and $\bar{V} \cap(X \backslash U)=\varnothing$. Applying Urysohn's Lemma, there exists a continuous function $\varphi: X \rightarrow[0,1]$ such that

$$
\varphi(x)=\left\{\begin{array}{l}
1, \quad x \in \bar{V} \\
0, \quad x \in X \backslash U .
\end{array}\right.
$$

Clearly, $\varphi$ is an observable. Choose $\varepsilon=\frac{1}{3} \int_{X} \varphi \mathrm{d} \mu>0$ and set

$$
D_{n}^{(\varepsilon)}=\left\{x \in X:\left|\frac{1}{n} \sum_{i=0}^{n-1} \varphi \circ T^{i}(x)-\int_{X} \varphi \mathrm{d} \mu\right|>\varepsilon\right\}, n=1,2, \ldots
$$

Since the pair $(T, \mu)$ satisfies LDT-III', one has

$$
\liminf _{n \rightarrow+\infty} \mu\left(D_{n}^{(\varepsilon)}\right)=0 .
$$

This implies that there exists an increasing sequence $\left\{N_{k}\right\}_{k=1}^{+\infty} \subset \mathbb{N}$ such that, for any $k \in \mathbb{N}$,

$$
\mu\left(D_{N_{k}}^{(\varepsilon)}\right) \leq \frac{\mu(U)}{2^{k+1}} .
$$

It follows from the choice of $\varphi$ that, for any $x \in U$,

$$
\left|\frac{1}{n} \sum_{i=0}^{n-1} \varphi \circ T^{i}(x)\right| \leq \frac{1}{n}\left|\left\{0 \leq i<n: T^{i}(x) \in U\right\}\right| \rightarrow 0,(n \rightarrow+\infty) .
$$


Thus,

$$
\lim _{n \rightarrow+\infty}\left|\frac{1}{n} \sum_{i=0}^{n-1} \varphi \circ T^{i}(x)-\int_{X} \varphi \mathrm{d} \mu\right|=3 \varepsilon .
$$

So, for any $x \in U$, there exists $M_{x} \in \mathbb{Z}^{+}$such that, for any $n \geq M_{x}, x \in D_{n}^{(\varepsilon)}$. This implies that, for any increasing sequence $\left\{n_{k}\right\}_{k=1}^{+\infty} \subset \mathbb{N}, U \subset \bigcup_{k=1}^{+\infty} D_{n_{k}}^{(\varepsilon)}$. Combining this with (5.1) yields that

$$
\mu(U) \leq \mu\left(\bigcup_{k=1}^{+\infty} D_{N_{k}}^{(\varepsilon)}\right) \leq \sum_{k=1}^{+\infty} \mu\left(D_{N_{k}}^{(\varepsilon)}\right) \leq \sum_{k=1}^{+\infty} \frac{\mu(U)}{2^{k+1}}=\frac{1}{2} \mu(U),
$$

which is a contradiction as $\mu(U)>0$.

(2) Suppose that there exist nonempty open subsets $U, V \subset X$ with $\mu(U) \mu(V)>0$ such that $N(U, V)=\varnothing$, i.e., for any $n \in \mathbb{Z}^{+}, T^{n}(U) \cap V=\varnothing$. Take $F_{1}=\overline{\cup_{n=0}^{+\infty} T^{n}(U)}$. Clearly, $F_{1} \subset X \backslash V$. Since the probability measure $\mu$ is regular and $\mu(V)>0$, there exists a closed subset $F_{2} \subset V$ such that $\mu\left(F_{2}\right)>0$. Noting that $F_{1} \cap F_{2}=\varnothing$, by Urysohn's Lemma, there exists a continuous function $\varphi: X \rightarrow[0,1]$ such that

$$
\varphi(x)= \begin{cases}1, & x \in F_{2}, \\ 0, & x \in F_{1}\end{cases}
$$

It is easy to see that for any $x \in U$ and any $i \in \mathbb{Z}^{+}, \varphi \circ T^{i}(x)=0$. So,

$$
U \subset \bigcap_{n=1}^{+\infty}\left\{x \in X:\left|\frac{1}{n} \sum_{i=0}^{n-1} \varphi \circ T^{i}(x)-\int_{X} \varphi \mathrm{d} \mu\right|>\frac{\mu\left(F_{2}\right)}{2}\right\},
$$

which is a contradiction as $(T, \mu)$ satisfies LDT-III' and $\mu(U)>0$.

Although we do not know if the probability measure $\mu$ is $T$-invariant when $(T, \mu)$ satisfies LDT-III or LDT-III', Corollary 5.1 below indicates that such a system admits a $T$-invariant probability measure $v \in M(X, T) \operatorname{such}$ that $\operatorname{supp}(v)=\operatorname{supp}(\mu)$.

Theorem 5.2. Let $(X, T)$ be a dynamical system and $\mu \in M(X)$. If $(T, \mu)$ satisfies LDT-III, then $(X, T)$ is an E-system. In particular, $T$ is $\mathscr{F}_{s}$-transitive.

Proof. Based on Theorem 5.1 and [39, Corollary 2.2], this holds trivially.

Corollary 5.1. Let $(X, T)$ be a dynamical system and $\mu \in M(X)$. If $(T, \mu)$ satisfies LDT-III', then $(\operatorname{supp}(\mu), T)$ is an E-system.

Lemma 5.1. Let $(X, T)$ be a dynamical system and $\mu \in M(X, T)$. If $(T, \mu)$ satisfies LDT-III', then $\mu$ is ergodic.

Proof. Given any fixed $\varphi \in C(X)$, the Birkhoff Ergodic Theorem implies that there exist $\varphi^{*} \in L^{1}(\mu)$ and $Y \in \mathcal{B}(X)$ with $\mu(Y)=1$ such that, for any $y \in Y, \lim _{n \rightarrow+\infty}(1 / n) \sum_{i=0}^{n-1} \varphi$ o $T^{i}(y)=\varphi^{*}(y)$. Since $(T, \mu)$ satisfies LDT-III', for any $N \in \mathbb{N}$ there exists an increasing sequence $\left\{L_{k}^{(N)}\right\}_{k=1}^{+\infty}$ such that, for any $k \in \mathbb{N}$,

$$
\mu\left(\Sigma\left(L_{k}^{(N)}, 1 / N\right)=\left\{x \in X:\left|\frac{1}{L_{k}^{(N)}} \sum_{i=0}^{L_{k}^{(N)}-1} \varphi \circ T^{i}(x)-\int_{X} \varphi \mathrm{d} \mu\right|>\frac{1}{N}\right\}\right) \leq \frac{1}{2^{k}}
$$


Set $\Sigma=\bigcap_{N=1}^{+\infty} \bigcup_{m=1}^{+\infty} \bigcap_{k=m}^{+\infty} X \backslash \Sigma\left(L_{k}^{(N)}, 1 / N\right)=X \backslash \bigcup_{N=1}^{+\infty} \bigcap_{m=1}^{+\infty} \bigcup_{k=m}^{+\infty} \Sigma\left(L_{k}^{(N)}, 1 / N\right)$, and take $\Omega=\Sigma \cap Y$. Then, $\mu(\Omega)=1$. According to the construction of $\Omega$, it follows that, for any $x \in \Omega$ and any $N \in \mathbb{N}$, there exists $m \in \mathbb{N}$ such that for any $k \geq m$,

$$
\left|\frac{1}{L_{k}^{(N)}} \sum_{i=0}^{L_{k}^{(N)}-1} \varphi \circ T^{i}(x)-\int_{X} \varphi \mathrm{d} \mu\right| \leq \frac{1}{N} .
$$

Combining this with $\lim _{k \rightarrow+\infty}\left(1 / L_{k}^{(N)}\right) \sum_{i=0}^{L_{k}^{(N)}-1} \varphi \circ T^{i}(y)=\varphi^{*}(x)$ yields that, for any $x \in \Omega$,

$$
\lim _{n \rightarrow+\infty} \frac{1}{n} \sum_{i=0}^{n-1} \varphi \circ T^{i}(x)=\varphi^{*}(x)=\int_{X} \varphi \mathrm{d} \mu .
$$

The proof is then completed by Lemma 3.1.

Theorem 5.3. Let $(X, T)$ be a dynamical system and $\mu \in M(X, T)$. Then, the following statements are equivalent:

(1) $(T, \mu)$ satisfies LDT-II'.

(2) $(T, \mu)$ satisfies LDT-III'.

(3) $\mu$ is ergodic.

Proof. They follow from Theorem 3.3 and Lemma 5.1.

Corollary 5.2. Let $(X, T)$ be a uniquely ergodic dynamical system and $\mu \in M(X)$. Then, the following statements are equivalent:

(1) $(T, \mu)$ satisfies LDT.

(2) $(T, \mu)$ satisfies LDT-I.

(3) $(T, \mu)$ satisfies LDT-II.

(4) $T$ is minimal and $\mu \in M(X, T)$.

(5) $(T, \mu)$ satisfies LDT-III and $\mu \in M(X, T)$.

Remark 3. Applying Theorem 5.2, it is not difficult to check that Theorem 4.2, Corollary 4.1, Corollary 4.2, and Corollary 4.3 all hold for LDT-III.

Although Theorem 5.3 proves that for a measure-preserving transformation, LDTII' is equivalent to LDT-III', we do not know if this holds for general transformations, because we can not answer the question as if the probability measure $\mu$ is $T$-invariant when $(T, \mu)$ satisfies LDT-III'. So, the following question is posed.

Question 1. Is there a transformation of a probability space $(X, \mathcal{B}(X), \mu)$, satisfying LDT-III' (resp., LDT-II'), which does not satisfy LDT-II' (resp., LDT-I')?

\section{A REMARK ON THE CENTRAL Limit THEOREM}

This section shows that, in contrast with the case of the large deviations theorem (see Corollary 5.2 and Remark 2), a dynamical system satisfying the Central Limit Theorem in the sense of Niu and Wang [25] does not exist.

Theorem 6.1. There exists no dynamical system satisfying the Central Limit Theorem in the sense of Niu and Wang. 
Proof. For any given dynamical system $(X, T)$, define an observable $\varphi: X \rightarrow \mathbb{C}$ as $\varphi(x) \equiv 0$. Take a probability measure $\mu \in M(X)$. For every interval $A \subset \mathbb{R}$, one has

$$
\begin{aligned}
& \lim _{n \rightarrow \infty} \mu\left(\left\{x \in X: \frac{1}{\sqrt{n}} \sum_{i=0}^{n-1}\left(\varphi \circ T^{i}(x)-\int_{X} \varphi \mathrm{d} \mu\right) \in A\right\}\right) \\
= & \mu(\{x \in X: 0 \in A\})=\left\{\begin{array}{ll}
1, & 0 \in A, \\
0, & 0 \notin A,
\end{array}=\mathbf{1}_{A}(0),\right.
\end{aligned}
$$

where $\mathbf{1}_{A}(\cdot)$ is the characteristic function of the set $A$. Clearly,

$$
\mathbf{1}_{A}(0)=\frac{1}{\sigma \sqrt{2 \pi}} \int_{A} e^{-t^{2} /\left(2 \sigma^{2}\right)} \mathrm{d} t
$$

does not hold for any interval $A \subset \mathbb{R}$. This implies that $\varphi$ does not satisfy the Central Limit Theorem in the sense of Niu and Wang. So, $(T, \mu)$ does not satisfy the Central Limit Theorem in the sense of Niu and Wang.

Remark 4. [32, Question 4.9] asks if there exists a dynamical system satisfying the Central Limit Theorem? Theorem 6.1 above shows that the answer is negative.

\section{REFERENCES}

1. C. Abraham, G. Biau, B. Cadre, Chaotic properties of a mapping on a probability space, J. Math. Anal. Appl., 266 (2002), 420-431.

2. E. Akin, S. Kolyada, Li-Yorke sensitivity, Nonlinearity, 16 (2003), 1421-1433.

3. J. Auslander, J.A. Yorke, Interval maps, factors of maps and chaos, Tôhoku Math. J., 32 (1980), 177-188.

4. V. Baladi, Positive transfer operators and decay of correlations. Advance series in nonlinear dynamics, vol. 16. World Scientific; 2000.

5. J. Banks, J. Brooks, G. Cairns, G. Davis, P. Stacey, On Devaney's definition of chaos, Amer. Math. Monthly, 99 (1992), 332-334.

6. L. Block, W.A. Coppel, Dynamics in one dimension. Lecture notes in mathematics, vol. 1513. Spring-Verlag; 1992.

7. R.L. Devaney, An Introduction to Chaotic Dynamical Systems. Redwood City: Addison-Wesley; 1989.

8. H. Furstenberg, Recurrence in Ergodic Theory and Combinatorial Number Theory. M. B. Porter Lectures, Princeton University Press, Princeton, N.J., 1981.

9. Conn., 1982), 131-148, Contemp. Math., 26, Amer. Math. Soc., Providence, R.I, 1984.

10. E. Glasner, B. Weiss, Locally equicontinuous dynamical systems, Colloq. Math., 84-85 (2000), 345-361.

11. R. Gu, The large deviations theorem and ergodicity, Chaos, Solitons \& Fractals, 34 (2007), 13871392.

12. L. He, X. Yan, L. Wang, Weak-mixing implies sensitive dependence, J. Math. Anal. Appl., 299 (2004), 300-304.

13. W. Huang, X. Ye, Homeomorphisms with the whole compacta being scrambled sets, Ergodic Theory and Dynamical Systems, 21 (2001), 77-91.

14. Devaney's chaos or 2-scattering implies Li-Yorke's chaos, Topology Appl., 117 (2002), 259-272.

15. $\ldots$ An explicit scateering, non-weakly mixing example and weak disjointness, Nonlinearity, 15 (2002), 849-862.

16. W. Huang, S. Kolyada, G. Zhang, Auslander-Yorke dichotomy theorem, multi-sensitivity and Lyapunov numbers, http: //arxiv .org/abs/1504.00587.

17. S. Kolyada, L. Snoha, Some aspect of topological transitivity—a survey, Grazer Math. Ber, Bericht Nr., 334 (1997), 3-35. 
18. S. Lardjane, On some stochastic properties in Devaney's chaos, Chaos, Solitons \& Fractals, 28 (2006), 668-672.

19. R. Li, The large deviations theorem and ergodic sensitivity, Commun. Nonlinear Sci. Numer. Simulat., 18 (2013), 819-825.

20. T.Y. Li, J.A. Yorke, Period three implies chaos, Amer. Math. Monthly, 82 (1975), 985-992.

21. C. Liverani, Decay of correlations, Ann. Math., 142 (1995), 239-301.

22. _ Decay of correlations for piecewise expanding maps, J. Stat. Phys., 78 (1995), 1111-1129.

23. T.K.S. Moothathu, Stronger forms of sensitivity for dynamical systems, Nonlinearity, 20 (2007), 2115-2126.

24. Y. Niu, The large deviations theorem and sensitivity, Chaos, Solitons \& Fractals, 42 (2009), 609614.

25. Y. Niu, Y. Wang, The central limit theorem and ergidicity, Statist. Probab. Lett., 80 (2010), 11801184.

26. J.C. Oxtoby, Ergodic sets, Bull. Amer. Math. Soc., 58 (1952), 116-136.

27. A.N. Sharkovsky, Coexistence of cycles of a continuous mapping of the line into itself, Ukrainian Math. J., 16 (1964), 61-71.

28. M. Viana, Stochastic dynamics of deterministic systems. Rio de Janeiro: Publications of CIMPA; 1997.

29. P. Walters, Some invariant $\sigma$-algebras for measure-preserving transformations, Trans. Amer. Math. Soc., 163 (1972), 357-368.

30. An Antroduction to Ergodic Theory. Springer-Verlag, New York, Heidelberg, Berlin; 1982.

31. C. Wu, Z. Xu, W. Lin, J. Ruan, Stochastic properties in Devaney's chaos, Chaos, Solitons \& Fractals, 23 (2005), 1195-1199.

32. X. Wu, G. Chen, Central limit theorem and chaoticity, Statist. Probab. Lett., 92 (2014), 137-142.

33. X. Wu, J. Wang, G. Chen, $\mathscr{F}$-sensitivity and multi-sensitivity of hyperspatial dynamical systems, J. Math. Anal. Appl., 429 (2015), 16-26.

34. X. Wu, G. Chen, On the large deviations theorem and ergodicity, Commun. Nonlinear Sci. Numer. Simulat. (In Press).

35. X. Wu, X. Wang, On the iteration properties of large deviations theorem, International Journal of Bifurcation and Chaos (In press).

36. X. Wu, P. Oprocha, G. Chen, On various definitions of shadowing with average error in tracing, http://arxiv.org/abs/1406.5822.

37. Z. Xu, W. Lin, J. Ruan, Decay of correlations implies chaos in the sense of Devaney, Chaos, Solitons \& Fractals, 22 (2004), 305-310.

38. X. Ye, R. Zhang, On sensitive sets in topological dynamics, Nonlinearity, 21 (2008), 1601-1620.

39. J. Yin, Z. Zhou, Positive upper density points and chaos, Acta Math. Sci. Ser. B Engl. Ed., 32 (2012), 1408-1414.

40. Y. Zhao, Some statistical results for Axiom A endomorphisms, Adv. Math. (China), 31 (2002), 200219.

(X. Wu) School of Sciences, Southwest Petroleum University, Chengdu, Sichuan, 610500, PeOple's RePublic of CHINA

E-mail address: wuxinxing5201314@163.com

(X. Wu;X. Wang) Institute for Advanced Study, ShenZhen University, Nanshan DisTRICT Shenzhen, GuAngdong, ChinA

E-mail address: wangxiong8686@szu.edu.cn

(G. Chen) Department of Electronic Engineering, City University of Hong Kong, Hong Kong SAR, PeOple's Republic of ChinA

E-mail address: eegchen@cityu.edu.hk 